\title{
Measurement of Hydrogen Diffusion through PVA Hydrogel using Magnetic Resonance Imaging Method in Comparison with Consistency Assessment using Digital Penetrometer
}

\author{
Dita Puspita Sari* and Darminto \\ Departement of Physics, Faculty of Mathematics and Natural Sciences, \\ Institut Teknologi Sepuluh Nopember (ITS), Kampus ITS Keputih Sukolilo, Surabaya 60111, Indonesia
}

\begin{abstract}
A study of hydrogen diffusion behavior in the PVA (polyvinyl-alcohol) hydrogel had been done to measure diffusion coefficient (Apparent Diffusion Coefficientl ADC) used as parameter of brain tumor grading. For our study, PVA hydrogel samples were prepared using freezing-thawing method with various concentration and number of freezing-thawing cycles. The ambiguity of brain tumor grading using ADC value, which is formed from Diffusion Weighted-Imaging 1,5 T, could be reduced enough with observing the result of correlation the ADC at b-value $1000 \mathrm{~s} / \mathrm{mm}^{2}$ and $3000 \mathrm{~s} / \mathrm{mm}^{2}$ with assessment of consistency measured using digital penetrometer based on microcontroller.
\end{abstract}

KEYWORDS: apparent diffusion coefficient, polyvinyl-alcohol, diffusion weighted imaging, digital penetrometer

\section{INTRODUCTION}

The fact that about two-thirds of the human body is made up of water, where each its molecule consists of two hydrogen atoms $\left({ }^{1} \mathrm{H}\right)$, of which makes MRI (Magnetic Resonance Imaging) possible. Diffusion-weighted magnetic resonance imaging (DW-MRI) is a highly sensitive tool for the detection of "Brownian motions", energized water molecules diffusing caused by heat associated with our body temperature, that characterize many brain pathologies including Brain Tumor.They are expressed, in diffusion weighted images, as changes in MRI signal intensity or as variations in the Apparent Diffusion Coefficient (ADC) of water [1,2]. Brain infraction leading to high mortality and disability, in recent years, has been emerged to be applicable in early diagnosis by DWMRI based on echo planar imaging (EPI) [3]. The measured signal intensity in the image with diffusion weighting is expressed by [4].

$$
\begin{aligned}
S(b) & =S_{\circ} \exp ^{-b(A D C)} \\
& =S_{\circ} \exp ^{--\gamma^{2} G^{2} \delta^{2}(\Delta-\delta / 3)(A D C)}
\end{aligned}
$$

with $\mathrm{S}(\mathrm{b})$ is the measured signal intensity in function of bvalue, the parameter $\gamma$ is a nuclear constant called gyromagnetic ratio, the parameter $\mathrm{G}$ is gradient pulses, the parameter $\delta$ is signal loss, the parameter $\Delta$ is time between the two pulses, and the ADC is the diffusion constant of water molecules.

DWI (Diffusion Weighted Imaging) and ADC value have

*E-MAIL: ditapuspitasari72@gmail.com been studied in other clinical situations, including the differential diagnosis and grading of brain tumors. It is known that the higher cellularity in high-grade glioma, the lower ADC values. But in some cases DWI at a standard $b$-value $(b=$ $1000 \mathrm{~s} / \mathrm{mm}^{2}$ ) at $1.5 \mathrm{~T}$ hardly differentiates high-grade and low-grade because of overlapping signal intensities $[S(b)]$ on DWI and ADC maps. Stronger gradients, recently, have permitted high b-value DWI particularly in 3T units which have higher SNRs compensating for it without increasing data acquisition time [5]. In this research, we prepared a sample to mimic the human tissue that would be scanned by DW-MRI $1.5 \mathrm{~T}$ at b-value $1000 \mathrm{~s} / \mathrm{mm}^{2}$ and $3000 \mathrm{~s} / \mathrm{mm}^{2}$.

There is a developing interest in the use of PVA cryogels to mimica range of tissue properties for MRI application, where the properties of the eminently resulting material can be exploited by the large effects of varying the concentration of PVA or the number of freezing-thawing cycle used in its preparation [6]. The suitable properties are including biocompatible, lack of toxicity due to chemical reaction, higher mechanical strength compared to the one formed by chemical method [7]. It has been described by the illustration in Figure 1 that the structure of PVA cryogels, which is a PVA hydrogels obtained by freezing-thawing (F-T) technique, consists in three phases; a water phase, amorphous phase and a crystalline phase [8]. According to recent experiment, increasing number of freezing-thawing cycles would increase the crystallite, the degree of physical crosslinking and rigidity of hydrogel, decrease water diffusion coefficient which is ADC in this research $[9,10]$.

On the other hand it has been assembled a digital microcontroller-based penetrometer by group of instrumentation physics department ITS [12]. The penetrometer provides kinetic energy which causes the penetrometer shaft to 


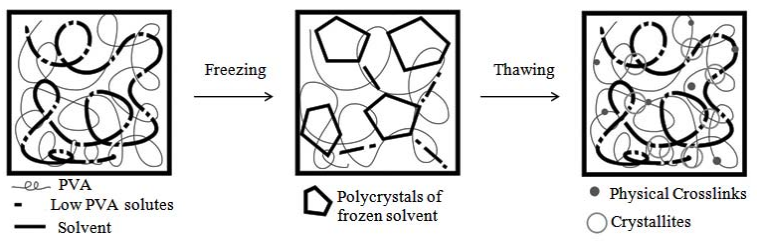

FIG. 1: From left to right; a low PVA concentration; the solvent crystals growth; the motion of amorphous PVA chains restricted by crystalline phase. It is a cryogelation process, gel formation via entanglement, hydrogen bonding and formation of crystallites. Reproduced from [11] adapted [8]]for cryogelation.

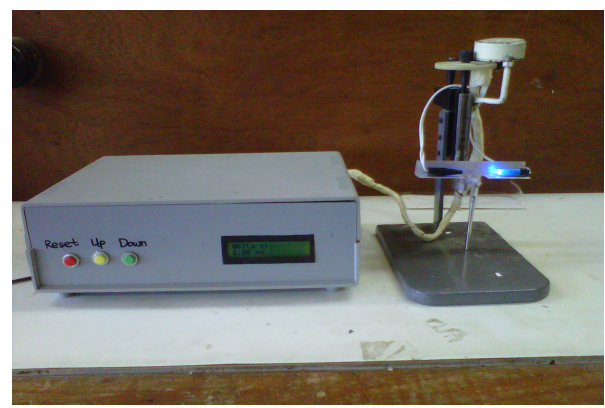

(a)

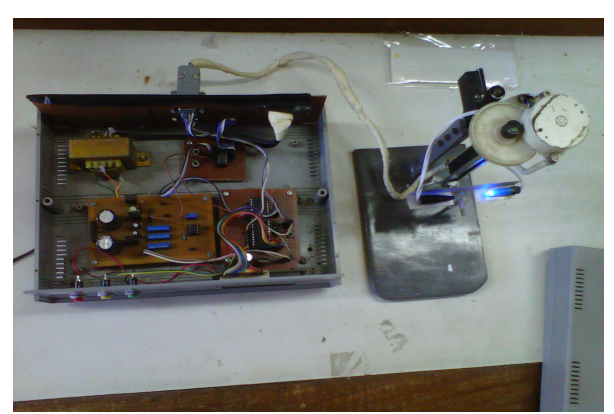

(b)

FIG. 2: From left to right; digital penetrometer based on microcontroller with reset, up, and down button; electrical design of digital penetrometer [12].

penetrate through the sample by mechanical properties, motor stepper. The sample resistance relating to the sample consistency will berestraining the tip of the digital penetrometer causing LED light is restricted by the needle, photodiode sensor will send a pulse to microcontroller ATMEGA16 causing the controlled motor stepper stops. In our experiment, we prepared PVA hydrogels with different concentration and number of freezing-thawing cycle. We measured the consistency of PVA sample using digital penetrometer and ADC value from DW-MRI $1.5 \mathrm{~T}$ at b-value $1000 \mathrm{~s} / \mathrm{mm}^{2}$ and $3000 \mathrm{~s} / \mathrm{mm}^{2}$. We would also observe the correlation between these two data, by using Pearson Correlation, in order to increase the assessment of brain tumor grading.

\section{MATERIAL AND METHOD}

\section{Hydrogel PVA Preparation}

In our experiment, PVA ( $\mathrm{Mw}=60,000$, degree of hydrolysis $98 \%$, Merck Schuchardt, Germany were used for PVA solution. According to a recent experiment result [13], PVA hydrogel samples were prepared by dissolving fully hydrolyzed PVA of 7,5, 10, 12,5, $15 \mathrm{wt} \%$ with deionized water (temperature $28,4^{\circ} \mathrm{C}$, salt $0 \mathrm{ppt}, \mathrm{pH} 7$, conductivity $0,01 \mu \mathrm{S}$ ), heating up $100^{\circ} \mathrm{C}$, in steady mixing which used hotplate stirrer Cimarec Digital-Thermolyne with magnetic stirring bars PTFE BRAND 137135 during an hour.The solutions were loaded into cylindrical plastic containers $(\phi 55 \mathrm{~mm} \times 95 \mathrm{~m})$ before cooling at room temperature for $3 \mathrm{~h}$. Cryogelation process were stimulated by doing two to fivefreezing-thawing cyclesat $-20^{\circ} \mathrm{C}$ for $12 \mathrm{~h}$ and $25^{\circ} \mathrm{C}$ for $12 \mathrm{~h}$.

\section{ADC Measurement using DW-MRI}

We used 1,5 T scanner (Signa Horizon; GE Medical System, Milwaukee, WI, USA) - standar head coil 8HBRAIN for Diffusion Weighted-MRI study. ADC value were gained from MRI with Diffusion-Weighted Imaging (DWI) method following Stejskal-Tanner sequence [13] and performed by using echo-planar imaging (EPI), FOV $24 \times 24 \mathrm{~cm}$, matrix size $512 \times 512$, section thickness $5 \mathrm{~mm}$, frequency 256 , scan time $1: 25, \mathrm{TR}=5000, \mathrm{TE}=92,4$ for b-value $1000 \mathrm{~s} / \mathrm{mm}^{2} ;$ dan TR $=5000, \mathrm{TE}=116$ for $\mathrm{b}=3000 \mathrm{~s} / \mathrm{mm}^{2}$.

ADC value was calculated by Functool software (GE Medical Systems) and obtained by calculating Signal Intensity (SI) on DWI after placing seven uniform region of interest (ROI)s in each sample. According to a recent experiment result [10], ADC value were calculated by averaging ADC values from several ROIs for each sample.

\section{Consistency Measurement using Digital Penetrometer}

We used in this experiment the digital penetrometer designed by Group of Instrumentation-Departement of Physics Institut Teknologi Sepuluh Nopember [14].

The samples were placing under digital penetrometer needle, which was a stainless steel blunt end cylinder $\phi 3 \mathrm{~mm} \times$ $50 \mathrm{~mm}$. The zero point of penetrometer was set to the samples, and then the data taken were seven rapidly different points for each sample. The depth of penetration was indicated the sample's consistency and would be correlated with the diffusion coefficient measured by DW-MRI.

\section{RESULT AND DISCUSSION}

The result from ADC measurement using different bvalue in DW-MRI for PVA hydrogel samples with different concentration and number of freezing-thawing cycle are described in Figure 3 and Figure 4. The result is analog 


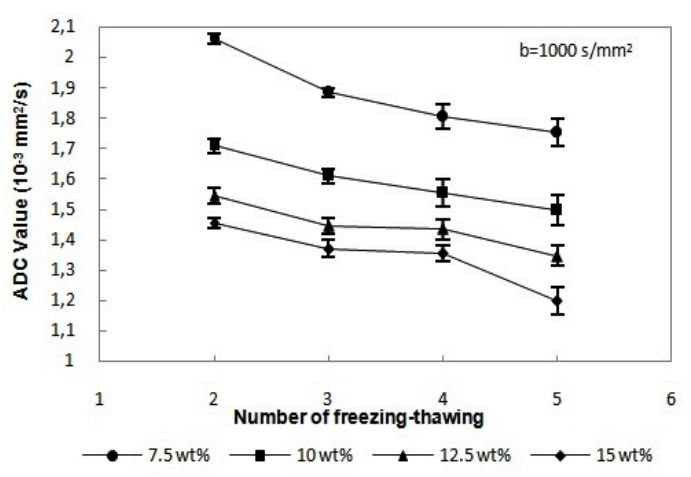

(a)

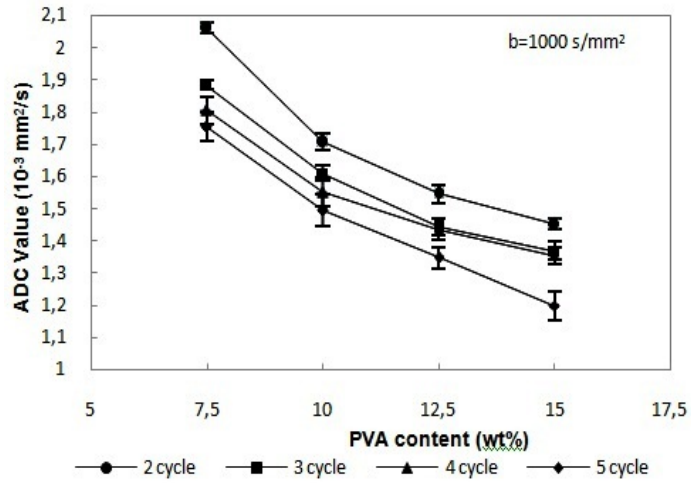

(b)

FIG. 3: From left to right; ADC values from PVA hydrogel at DW-MRI $b=1000 \mathrm{~s} / \mathrm{mm}^{2}$ as function of freezing-thawing cycle at different PVA concentration; ADC values from PVA hydrogel at DW-MRI $b=3000 \mathrm{~s} / \mathrm{mm}^{2}$ as a function of PVA concentration at different number of freezing-thawing.

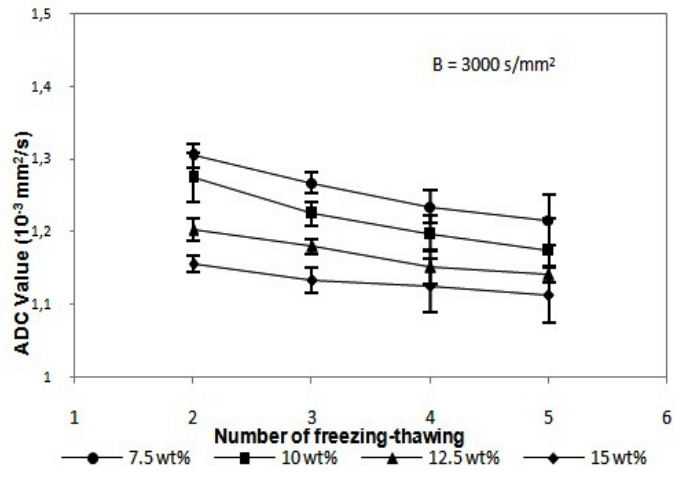

(a)

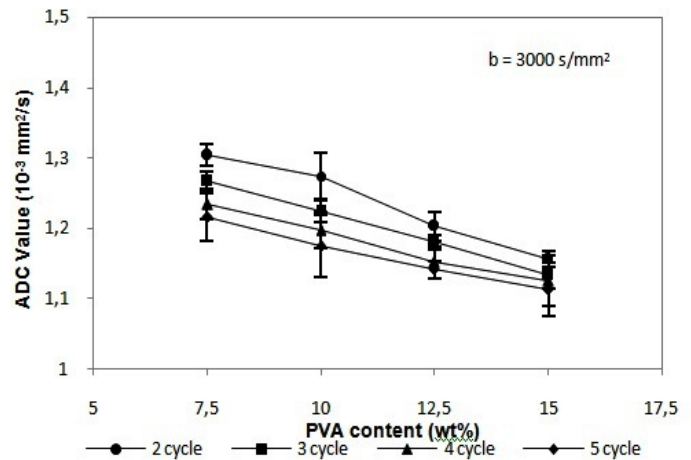

(b)

FIG. 4: From left to right; ADC values from PVA hydrogel at DW-MRI $b=3000 \mathrm{~s} / \mathrm{mm}^{2}$ as function of freezing-thawing cycle at different PVA concentration; ADC values from PVA hydrogel at DW-MRI $b=3000 \mathrm{~s} / \mathrm{mm}^{2}$ as a function of PVA concentration at different number of freezing-thawing.

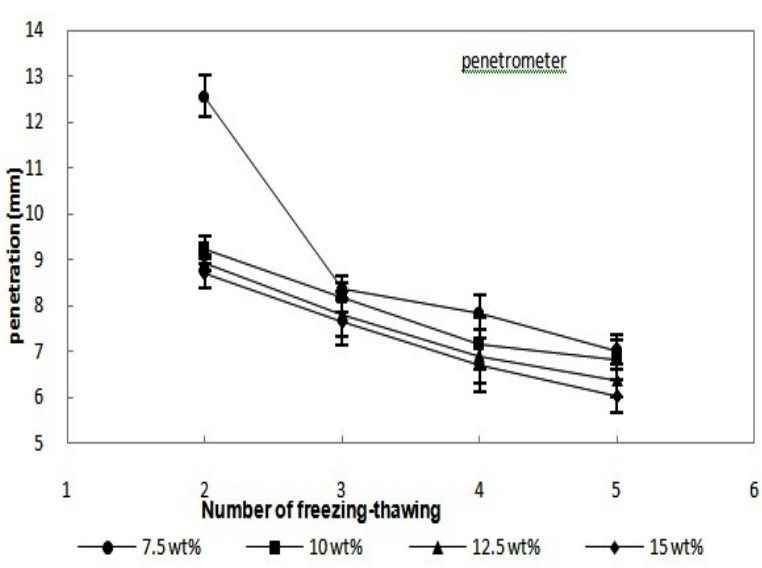

(a)

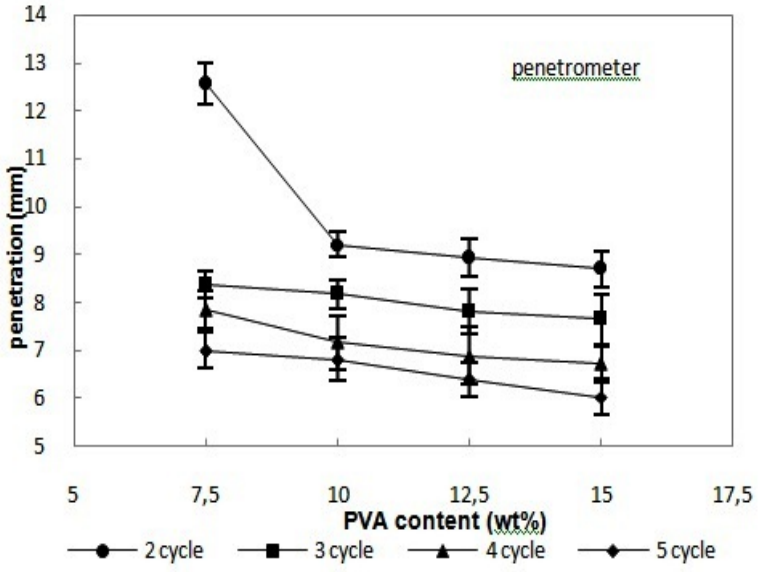

(b)

FIG. 5: From left to right; Consistency from digital penetrometer measurement as a function of number of freezing-thawing cycle at different PVA concentration; Consistency from digital penetrometer measurement as a function of PVA concentration at different number of freezingthawing cycle. 
TABLE I: Pearson Correlation of two data.

\begin{tabular}{ccccccccc}
\hline \hline Data & 2 cycles & 3 cycles & 4 cycles & 5 cycles & $7.5 \mathrm{wt} \%$ & $10 \mathrm{wt} \%$ & $12.5 \mathrm{wt} \%$ & $15 \mathrm{wt} \%$ \\
\hline $\mathrm{b}=1000$ & 0.96 & 0.96 & 0.99 & 0.96 & 0.98 & 0.99 & 0.94 & 0.92 \\
$\mathrm{~b}=3000$ & 0.77 & 0.99 & 0.96 & 0.97 & 0.93 & 0.99 & 0.99 & 0.98 \\
\hline \hline
\end{tabular}

with our hypothesis of higher diffusion coefficient with lower concentration and lower number of freezing and thawing $[10,15]$. In Figure 4, it is showed that the linearity of the ADC value graph at b-value $3000 \mathrm{~s} / \mathrm{mm}^{2}$ is better than ADC graph at b-value $1000 \mathrm{~s} / \mathrm{mm}^{2}$ which is in line with the sample parameter that we used.

The consistency values from digital penetrometer measurement are showed by Figure 5. In the Table I, it is showed that both data at b-value $1000 \mathrm{~s} / \mathrm{mm}^{2}$ and b-value 3000 $\mathrm{s} / \mathrm{mm}^{2}$ have good correlation with physical measurement of consistency assessment using digital penetrometer.

\section{CONCLUSION}

A study of hydrogen diffusion behavior in the PVA hydrogel to measure diffusion coefficient (Apparent Diffusion Coefficient) used as parameter of brain tumor grading had been done. A bare differentiating of brain tumor grading, by applying conventional DW-MRI $1.5 \mathrm{~T}$ b value $1000 \mathrm{~s} / \mathrm{mm}^{2}$, can be reduced by adding other parameter which is b-value 3000 $\mathrm{s} / \mathrm{mm}^{2}$. However higher b-value at conventional DW-MRI 1.5 $\mathrm{T}$ are not usually used in clinical practice because of poor image quality, so both of b-value $1000 \mathrm{~s} / \mathrm{mm}^{2}$ and higher are recommended for brain tumor grading.
[1] L. Tonarelli, ECEI. (810) 229-3354 (2012).

[2] T. Moritani, et al., Diffusion-Weighted MR Imaging of the Brain (Springer-Verlag Berlin Heidelberg, 2009).

[3] Shen et al., BMC Medical Imaging 11:2 (2011).

[4] S. Mori, and P. B. Barker, The Anatomical Record (New Ana T.) 257, 102-109 (1999).

[5] H.S. Seo, et al., AJNR 29 (2008)

[6] D.M. King, et al., Ultrasound Med Biol. 37(5), 813-26./ j.ultrasmedbio.02.012 (2011)

[7] V.I. Lozinsky, Polymeric Cyogels as Promising Materials of Biotechnological Interest (Elsevier Publishing, 2003)

[8] Y. Dwihapsari, et al., JFA, 6 (2) (2010).

[9] P.J. R-Hernandez, et al., Journal of Magnetism and Magnetic Materials, 329, e373-e376 (2008)

[10] Y. Dwihapsari, et al., The Assessment of Consistency using Penetrometer and Apparent Diffusion Coefficient (ADC) Value using Diffusion Weighted Magnetic Resonance Imaging (DW-MRI) from Polyvinyl Alcohol (PVA) Formed by Freezing-Thawing Cy- cle, AIP Conf. Proc. Bandung, Indonesia, 2012.

[11] N.E. Vrana, Use of Poly Vinyl Alcohol (PVA) Cryogelation for Tissue Engineering: Composites Scaffold Formation and Cell Encapsulation, Thesis presented for the degree of $\mathrm{PhD}$, Dublin City University, Ireland, 2009.

[12] E.O. Stejskal, and J.E. Tabber, The Journal of Chemical Physics 42, 288-293 (1965).

[13] D.P. Sari, Study of Apparent Diffusion Coefficient at Magnetic Resonance Imaging for PVA Hidrogel and Its Correlation with Consistency Measurement using Penetrometer, Final Project, Institut Teknologi Sepuluh Nopemeber, Surabaya, 2011.

[14] E.P. Suwanto, Study and Design of Digital Penetrometer for Material Consistency Testing Based on Microcontroller, Final Project, Institut Teknologi Sepuluh Nopember, Surabaya, 2011.

[15] C.M. Hassan, and N.A. Peppas, Advances in Polymer Science, 153 (2000). 